\title{
Efecto sobre el sistema de la coagulación del zumo de frutas y hortalizas peruanas
}

\author{
Edwin Zarzosa-Norabuena ${ }^{1,2}$, Berta Loja-Herrera ${ }^{1,3}$, Alberto Salazar-Granara ${ }^{1,4}$, Miguel Inocente-Camones ${ }^{1,5}$.
}

\section{RESUMEN}

Objetivo: Comprobar el efecto anticoagulante in vitro e in vivo de frutas y hortalizas peruanas e identificar la vía de la coagulación sobre la que actúa.

Material y Métodos: Se extrajo los zumos de Ananascomosus (L.) Merr, Citrus limonun Risso, Carica papaya L., Alliumsativum L., Allium cepa L. y Zingiber officinale L. Roscoe. Se realizó un estudio in vitro con plasma humano e in vivo administrando el zumo vía oral a ratas por catorce días. La actividad anticoagulante fue evaluada midiendo el tiempo de protrombina (TP) y el tiempo de tromboplastina parcial activado (TTPa), además se determinó el fibrinógeno.

Resultados: In vitro los zumos de Ananas y Zingiber prolongaron el TP, Carica el TTPa y los de Citrus, Alliumsativumy cepa ambas pruebas. In vivo los zumos de Allium cepa y Carica prolongaron el TP, Citrus el TTPa, Ananas ambas pruebas. Los zumos de Ananas, Citrus, Allium sativum y Zingiber disminuyeron el fibrinógeno.

Conclusión: In vitro e in vivo los zumos de Ananascomosus, Citrus limonun, Carica papaya y Allium cepa tienen efecto anticoagulante. Los zumos de Allium sativum y Zingiber officinale, tienen este efecto solo in vitro.

(Horiz Med 2015; 15(2): 6-11)

Palabras clave: Frutas, hortalizas, sistema coagulación, Carica papaya, Ananascomosus, Citrus limón, Carica papaya, Allium cepa, Allium sativum, Zingiber officinale. (Fuente: DeCS BIREME).

\section{Effect on the clotting system of Peruvian fruit and vegetable juice}

\section{ABSTRACT}

Objective: Verify the anticoagulant effect in vitro and in vivo of Peruvian fruit and vegetable juice and identify the coagulation pathway on which it acts.

Material and Methods: Extracted juices of Ananascomosus (L.) Merr, Citrus limonunRisso, Carica papaya L., Allium sativum L., Allium cepa L. and Zingiber officinale L. Roscoe. An in vitro study with human plasma and in vivo administering juice orally to rats for 14 days. Anticoagulant activity was evaluated by measuring the prothrombin time (PT) and partial activated thromboplastin time (APTT), the Fibrinogen was also determined.

Results: In vitro juices of Ananas and Zingiber prolonged PT, Carica prolonged APTT and Citrus, Allium sativum and cepa prolonged both tests. In vivo, juices of Allium cepa and Carica prolonged PT, citrus prolonged APTT, and Anannas both tests. The juices of Anannas, Citrus, Allium sativum and Zingiber decreased Fibrinogen.

Conclusion: In vitro and in vivo Ananascomosus, Citrus limonun, Carica papaya and Allium cepa juices have anticoagulant effect. The juices of Allium sativum and Zingiber officinale, have this effect only in vitro. (Horiz Med 2015; 15(2): 6-11)

Key words: fruits, vegetables, system, coagulation, Carica papaya, Ananas, Citrus lemon, Carica papaya, Allium cepa, Allium sativum, Zingiber officinale. (Source: MeSH NLM).

\footnotetext{
Centro de Investigación de Bioquímica y Nutrición. Instituto de Investigación. Facultad de Medicina Humana. Universidad de San Martin de Porres. Lima, Perú.

2. Tecnólogo médico. Magister en Bioquímica y Nutrición.

Bióloga. Doctora en Ciencias Biológicas.

Médico Cirujano. Doctor en Medicina.

Químico Farmaceutico. Magister en Productos Naturales y Biocomercio.
} 


\section{INTRODUCCIÓN}

Las enfermedades cardiovasculares (ECV) son la principal causa de mortalidad en el mundo. En el Perú, en el año 2012 las enfermedades isquémicas del corazón y las cerebrovasculares ocuparon la quinta y sétima causas de muerte respectivamente, con más de 8 mil casos anuales (1).

Evidencias epidemiológicas indican que una dieta rica en frutas y hortalizas, como la dieta mediterránea, promueve la salud, disminuyendo el riesgo a desarrollar enfermedades cardiovasculares $(2,3)$.Esto se debería a su aporte en nutrientes, nutracéuticos, antioxidantes y fibras.

El efecto antitrombótico (antiagregante plaquetario, anticoagulante y fibrinolitico) de frutas y hortalizas es poco conocido (4).

Investigaciones realizadas han demostrado que Ananascomosus, Vitisvinifera,Rubusidaeus, Alliums ativum y Allium cepa, cultivadas en otras regiones, tienen efecto anticoagulante $(4,5)$.

La gastronomía peruana utiliza una variedad de frutas y hortalizas, además el Perú se ha convertido en exportador líder a nivel internacional de estos productos (6). Sin embargo; no existen estudios peruanos sobre su efecto antitrombótico.

El propósito de este estudio es comprobar la actividad anticoagulante in vitro e in vivo de frutas y hortalizas peruanas e identificar la vía de la coagulación sobre la que actúa.

\section{MATERIAL Y MÉTODOS}

Tipo de estudio: analítico, transversal, experimental y prospectivo.

\section{Muestra vegetal}

Las especies fueron adquiridas de los cultivos de la Universidad Nacional Agraria La Molina - Lima (UNALM), y fueron los frutos: Ananascomosus (L.) Merr (piña), Citrus limonun Risso (limón) y Caricapapaya L.(papaya), y las hortalizas:Allium sativum L.(ajo), Allium cepa L.(cebolla), y Zingiber officinale $\mathrm{L}$.
Roscoe (jengibre o kión). La determinación taxonómica estuvo a cargo de una especialista en Botánica de la FMH-USMP.

\section{Preparación del zumo}

Las frutas y hortalizas seleccionadas fueron lavadas, peladas y cortadas en trozos pequeños y mediante una prensa manual se procedió a extraer el zumo, el cual fue filtrado, en coffe filters $\mathrm{N}^{\circ} 2$. Los zumos se prepararon cada día de experimentación.

\section{Muestra biológica}

Se extrajo sangre venosa en tubos de $4.5 \mathrm{~mL}$ con 0,5 $\mathrm{mL}$ con citrato de sodio al $3,2 \%$, en cinco voluntarios sanos, con edades entre 19 a 25 años, que no recibían medicación y con índice de masa corporal normal, los cuales otorgaron su consentimiento informado. Se centrifugó la muestra a 3000 r.p.m. por diez minutos y se separó el plasma.

\section{Muestra animal}

Se utilizaron ratas albinas de la línea Holtzman, de ambos sexos, obtenidos del Bioterio de la Facultad de Zootecnia (UNALM), con un peso promedio de 150 a 200 gramos.

Los animales fueron acondicionados en el Bioterio de la FMH-USMP, durante siete días antes del tratamiento; se separaron y colocaron en jaulas amplias, provistos de alimento equilibrado y agua ad libitum. Se siguió las guías de manejo y cuidado de animales de laboratorio (7).

\section{Pruebas de coagulación}

Se procesaron en un equipo coagulométrico Stat compact, que usa un método electromagnético, utilizando kit de reactivos de Diagnostica Stago:

Los resultados de tiempo de protrombina (TP) y tiempo de tromboplastina parcial activado (TTPa), se expresan en segundos(s) y el fibrinogeno en $\mathrm{mg} / \mathrm{dl}$.

\section{Evaluación del efecto anticoagulante in vitro}

Se adicionó $2,9 \mathrm{~mL}$ de plasma, a siete tubos, al primero se colocó $0,1 \mathrm{~mL}$ de suero fisiológico (grupo control), y a los siguientes tubos $0,1 \mathrm{~mL}$ de cada uno de los zumos preparados.

Se incubó cinco minutos a $37^{\circ} \mathrm{C}$ y se realizaron las mediciones de TP y TTPa. 


\section{Evaluación del efecto anticoagulante in vivo}

Se dividieron en siete grupos de seis ratas en cada grupo, diariamente se administró por via oral en las mañanas, utilizando una cánula orogástrica, las siguientes dosis:

Grupo I (control): $2 \mathrm{~mL} / \mathrm{kg}$ de peso, de solución fisiológica.

Grupo II a IV: $2 \mathrm{~mL} / \mathrm{kg}$ de peso de zumo de A. comosus, C. limonun y C. papaya respectivamente. Grupo V a VIl: $1 \mathrm{~mL} / \mathrm{kg}$ de peso de zumo de A. sativum, A. cepa y Z.officinale, respectivamente. El día catorce en ayuno de 14 horas, se anestesió a las ratas con pentobarbital sódico vía intraperitoneal y se extrajo1,5 $\mathrm{mL}$ de sangre, por punción intracardiaca en un tubo con citrato de sódio, para determinar TP, TTPa y fibrinógeno $(7,8)$.

\section{Análisis estadístico}

Los datos cuantitativos se expresaron en medias y desviación estándar. La evaluación de la significancia estadística se realizó mediante la prueba t de Student. El nível de significancia se fijó en una probabilidad menor 0 igual que 0,05 y a un intervalo de confianza del $95 \%$.

\section{RESULTADOS}

\section{Estudio in vitro}

En la Tabla $\mathrm{N}^{\circ} 1$, puede observarse que el TP fue considerablemente mayor para los zumos de C. limonuny A. cepa, con 105.9 s y 82.7 s respectivamente, los otros zumos mostraron valores ligeramente más elevados que el grupo control. Los valores más altos de TTPa correspondieron a C. limonun y A. cepa con $143.2 \mathrm{~s}$ y $104.6 \mathrm{~s}$ respectivamente, en cambio el $A$. comosus tuvo un valor menor que el control, mientras que $C$. papaya, A. sativum y $Z$. officinale tuvieron valores estadísticamente significativos que el control.

\section{Estudio in vivo}

En la Tabla $N^{\circ} 2$, se observa que el TP es más elevado que el grupo control, en aquellas ratas tratadas con A. comosus, C. papaya y A. cepa, mientras que las que recibieron zumo de C. limonun, $C$. sativum y $Z$. officinale mostraron valores ligeramente mayores
Tabla 1. Resultados de las pruebas de TP y TPTA, in vitro.

\begin{tabular}{|c|c|c|}
\hline GRUPOS & TP (S) & TTPa (S) \\
\hline Control & $13.4 \pm 0.5^{*}$ & $39.5 \pm 3.3$ \\
\hline A. comosus & $16.5 \pm 0.8^{*}$ & $35.3 \pm 3.9$ \\
\hline C. limonun & $105.9 \pm 55.4^{*}$ & $143.2 \pm 51.9^{*}$ \\
\hline C. papaya & $14.1 \pm 0.8$ & $98.2 \pm 6.2^{*}$ \\
\hline A. sativum & $14.6 \pm 0.7^{*}$ & $51.2 \pm 3.3^{*}$ \\
\hline A. cepa & $82.7 \pm 33.9^{*}$ & $104.6 \pm 29.7^{*}$ \\
\hline Z. officinale & $14.5 \pm 0.9^{*}$ & $49.1 \pm 5.7^{*}$ \\
\hline${ }^{*} p \leq 0.05$ & & \\
\hline
\end{tabular}

Tabla $\mathbf{N}^{\circ}$ 2. Resultados de las pruebas de TP, TPTa y fibrinógeno, in vivo.

$\begin{array}{lccc}\text { GRUPOS } & \text { TP (S) } & \text { TTPa (S) } & \begin{array}{c}\text { Fibrinógeno } \\ \text { (mg/dl) }\end{array} \\ \text { Control } & 16.1 \pm 0.5^{*} & 23.5 \pm 3.3 & 237 \pm 19 \\ \text { A. comosus } & 17.3 \pm 0.7^{*} & 27.2 \pm 2.3 & 211 \pm 6^{*} \\ \text { C. limonun } & 16.7 \pm 0.8^{*} & 26.8 \pm 1.8^{*} & 209 \pm 6^{*} \\ \text { C. papaya } & 17.5 \pm 0.1 .0^{*} & 26.2 \pm 2.2^{*} & 261 \pm 6.2^{*} \\ \text { A. sativum } & 16.8 \pm 0.7^{*} & 19.9 \pm 3.38^{*} & 217 \pm 18^{*} \\ & & & \\ \text { A. cepa } & 18.1 \pm 0.8^{*} & 26.1 \pm 2.6^{*} & 205 \pm 11^{*} \\ & & & \\ \text { Z. officinale } & 15.9 \pm 1.1^{*} & 22.5 \pm 3.0^{*} & 188 \pm 25^{*} \\ & & & \end{array}$


que el control, sin significancia estadística. Los valores de TTPa fueron mayores para A. comosus y $\mathrm{C}$. limonun, los otros grupos no mostraron diferencia con el control. Al evaluar los niveles de fibrinógeno se observó que el grupo de ratas que recibió el zumo de C. papaya, tuvo un valor más elevado que el control, mientras que al que se administró $A$. sativum no se observó variación alguna.

Aquellas que recibieron zumo de A. comosus, C. limonun y $Z$. officinale mostraron menores valores que el control.

\section{DISCUSIÓN}

El consumo regular de frutas y hortalizas disminuye el riesgo cardiovascular, por lo que ha causado interés en conocer los componentes bioactivos y los mecanismos involucrados (9).

Uno de los posibles mecanismos seria el efecto anticoagulante que poseen. La coagulación sanguínea es un complejo proceso de reacciones proteolíticas en cadena, con dos vías, la intrínseca (factores VIII, IX y XI) y la extrínseca (factores II, V, VII y X), siendo evaluadas por las pruebas de laboratorio, tiempo de tromboplastina parcial activada (TTPa) y tiempo de protrombina (TP) respectivamente, detectando la deficiencia de alguno de estos factores (10).

Dentro de las frutas se ha reportado que la Ananascomosus tiene actividad anticoagulante, su extracto acuoso contiene enzimas proteolíticas, denominada bromelina, que de acuerdo a estudios in vitro y en animales prolonga el TP y TTPa (11), el cual concuerda con nuestros resultados, donde vimos que el zumo tiene el mismo efecto in vitro sobre el TP y en ratas en ambas pruebas. Lo que indica que in vivo el zumo de Ananascomosus tiene efecto sobre ambas vías de la coagulación, el probable mecanismo seria por reducción de los aceleradores del inicio de la vía intrínseca y la disminución del factor X (Stuart) y II (protrombina), factores de la vía extrínseca (12).

El Citrus limonun contiene muchos micronutrientes como el potasio, vitamina $\mathrm{C}$, folatos y flavonoides. In vitro, el zumo aumentó el TTPa pero no al TP (13), en este estudio alteró ambas pruebas.
En investigaciones en ratas la ingesta oral del zumo aumentó el TP (14) y en conejos no alteró estas pruebas (13), en este estudio aumentó el TTPa, es decir actúa sobre la vía intrínseca, estas diferencias en los resultados quizás se deberían a las distintas variedades de Citrus utilizadas y a la procedencia geográfica.

Está documentada la interacción entre el consumo del extracto de Carica papaya L.y el anticoagulante oral warfarina, afectando los valores de TP, este fruto contiene la enzima proteolítica papaína (15), en este estudio el zumo alteró in vitro el TTPa e in vivo el TP, afectando la vía extrínseca.

Las especies Allium, especialmente sativum y cepa, contienen compuestos órgano-sulfurados con actividad anticoagulante, por ejemplo el ajo en 0 en el sativum $(16,17)$.

El Allium sativum, es ampliamente usada tanto en la alta culinaria como en los platos típicos de muchas regiones del mundo. Estudios in vitro indican que el aceite altera el TP (18), en este estudio el zumo prolongo el TP y TTPa. En ratas con una dieta alta en colesterol, tratadas con el extracto acuoso, por cuatro semanas prolonga ambas pruebas (19), en este estudio donde la dieta fue equilibrada, no hubo variación de estas pruebas.

El Allium cepa, se usa como condimento, alimento fresco o encurtido. In vitro el zumo aumentó el TP y TTPa, concordante con otros trabajos donde el extracto acuoso aumentó el TP (18). En las ratas aumentó el TP, contrario a un estudio donde la suplementación con extracto etanólico no afecto las pruebas de coagulación (20). No todas las variedades de Allium cepa presentan este efecto anticoagulante, por ello los resultados contradictorios (21).

El zumo de los rizomas de Zingiber officinale, in vitro prolongó el TP y TTPa. En las ratas no hubo alteración de estas pruebas, resultados similares a otras investigaciones donde se administró a ratas el jugo (22) y el extracto etanólico (23), sin embargo se ha reportado casos de interacción en pacientes que recibieron warfarina (15). 
El fibrinógeno es una proteína del sistema de la coagulación, su aumento representa un factor de riesgo cardiovascular independiente (24). Los zumos de Ananas, Citrus, A. cepa y Zingiber disminuyeron significativamente el fibrinógeno, lo que concuerda con otros estudios $(11,13)$.

Se debería promover el consumo de frutas y hortalizas, con el valor agregado de sus propiedades antitromboticas. Su consumo en la población peruana es menor a la recomendada por la Organización Mundial de la Salud, que es de cinco o más raciones diarias (25).

Por último; debe realizarse más estudios en diversas variedades de frutas y hortalizas para conocer el mecanismo de acción de este efecto anticoagulante e identificar los principios activos de dicha actividad.

En conclusión, In vitro e in vivo los zumos de Ananascomosus, Citrus limonun, Carica papaya y Allium cepa tienen efecto sobre la vía intrínseca y/o extrínseca de la coagulación. Los zumos de Alliumsativum y Zingiber officinale, tienen este efecto sobre ambas vías, solo in vitro.

\section{REFERENCIAS BIBLIOGRÁFICAS}

1. Ministerio de Salud Perú. Análisis de la situación de salud (ASIS) 2012.

2. Bazzano $L$, et al. Fruit and vegetable intake and risk of cardiovascular disease in US adults: the first National Health and Nutrition Examination Survey Epidemiologic Follow-up Study. Am J Clin Nutr. 2002;76(1): 93-9.

3. Ramon E et al. Primary Prevention of Cardiovascular Disease with a Mediterranean Diet. New England Journal of Medicine 2013; 368: 1279-90.

4. Torres C, Guzmán L, Moore R, Palomo I. Efecto antitrombótico, una característica poco conocida de las frutas y hortalizas. Revista Chilena de nutrición 2008; 35: 10-17.

5. Torres C, et al. Antiplatelet, anticoagulant, and fibrinolytic activity in vitro of extracts from selected fruits and vegetables. Blood coagul Fibrinolysis. 2011; 22 (3): 197-205.
6. Centro Nacional de Planeamiento estratégico. Gastronomía peruana al 2021. 2da. Edición. Lima: 2012.

7. Franklin C, Suckow M, Weisbroth S. The Laboratory rat. 2da edición. New York: Elsevier, editor, 2006.

8. Vogel G. Pharmacologic assays in thrombosis and haemostasis. En Drug Discovery and evaluation Pharmacological Assay. 2da. edición. Ed. Springer. 2002. Alemania. 394-5.

9. Palomo I, et al. El consumo de frutas y hortalizas ayuda a prevenir el daño endotelial. Revista chilena de nutrición 2011; 38 (3): 343-55.

10. Palomo I, et al. Estudio de laboratorio de las enfermedades hemorragiparas. En Hematología, fisiopatología y diagnóstico. Ed. Universidad de Talca. Chile 2005. 745-52.

11. Maurer H. Bromelain: biochemistry, pharmacology and medical use. Cell Mol Life Sci. 2001; 58(9):1234-45.

12. Pavan R, Jain S, Shraddha, Kumar A. Properties and therapeutic application of bromelain: a review. Biotechnology Research International 2012; 2012: 1-5.

13. Riaz A et al. In vitro/in vivo effect of Citrus limón juice on blood parameters, coagulation and anticoagulation factors in rabbits. J Pharm Sci. 2014; 27(4): 907-15.

14. Adepoju G, Adeyemi T. Evaluation of the effect of lime fruit juice on the anticoagulant effect of warfarin.J Young Pharm. 2010;2 (3): 269-72.

15. Nutescu E, Shapiro N, Ibrahim S, West P. Warfarin and its interactions with foods, herbs and other dietary supplements. Expert Opin Drug Saf. 2006; 5(3): 433-51.

16. Corzo M, Corzo N, Villamiel M. Biological properties of onion and garlic. Trends in Food Science \& Technology 2007;18 (12): 609-625

17. García L, Sánchez F. Revisión: efectos cardiovasculares del ajo (Alliumsativum). Archivos Latinoamericanos de Nutrición 2000; 50 (3): 219-29.

18. Mansoor N. In vitro study of the anticoagulant activity of some plant extracts. Indian Journal of applied research 2013; 3 (7): $120-22$.

19. Alhamami $\mathrm{O}$ et al. Effects of garlic on haemostatic parameters and lipid prolife in hyperlipidemic rats: antitherogenic and 
antithrombotic effects. Eastern Journal of Medicine 2006; 11: 13-18.

20. Lee SM, Moon J, Chung JH, Cha YJ, Shin MJ. Effect of quercetin-rich onion peel extracts on arterial thrombosis in rats. Food and Chemical.Toxicology 2013; 57: 99-105.

21. Yamada $\mathrm{K}$, et al. An onion variety has natural antithrombotic effect asassessed by thrombosis/thrombolysis models in rodents. Thromb Res 2004; 114(3): 213-20.

22. Prasad et al. To investigate the action of ginger-juice zengiberofficinale roscoe on blood coagulation process. International Journal of Pharma Sciences and Research 2012; 3 (7): 407-15.

23. Weidner $M$, Sigwart $K$. The safety of a ginger extract in the rat. Journal of Ethnopharmacology 2000; 73: 513-20.

24. Borissoff JI, Spronk HM, Cate H. The hemostatic system as a modulator of atherosclerosis. New England Journal of Medicine 2011; 364(18): 1746-60

25. Instituto Nacional de Estadísticas e Informática. Perú enfermedades no transmisibles y transmisibles, 2013.

\section{Agradecimientos}

A Glorinda Quispe, Oscar Mishti y Carlos Pante por su apoyo técnico en el trabajo de investigación.

\section{Correspondencia:}

\section{Fuentes de Financiamiento \\ Financiado por la Universidad de San Martin de Porres.}

\section{Conflictos de interés}

Los autores declaran no tener conflictos de interés.
Edwin Zarzosa Norabuena

Dirección: Av. Alameda del Corregidor 1531. La Molina. Lima, Perú

Teléfono: (511)365 2300

Correo electrónico: ezarzosan@usmp.pe 